\title{
un nouvel essai pour la mesure de la résistance à la traction
}

\section{a new test for tensile strength measurements}

\author{
M.P. LUONG \\ Laboratoire de Mécanique des Solides \\ C.N.R.S. UA $317^{*}$
}

\section{Résumé}

Un nouvel essai de traction homogène a été mis au point au Laboratoire de Mécanique des Solides. Sa réalisation est simple, rapide et économique. Son principe est théoriquement très satisfaisant.

Son application aux roches, aux bétons, aux céramiques, etc., est particulièrement commode dans des conditions d'environnements variés, voire hostiles.

\section{Abstract}

A new homogeneous traction test has been deviced at the Laboratoire de Mécanique des Solides. This experimental technique is simple, practical and reliable as well as rigorous from a mechanics point of view.

It provides a simple means for obtaining the tensile strength of rock as well as concrete, ceramics, and so on... under various conditions of loading and even in hostile environment. 


\section{INTRODUCTION}

La résistance à la traction est un excellent indice de qualité des matériaux fragiles comme les roches ou les bétons. La rupture se produit suivant le mode I de la Mécanique de la Rupture, le plus dangereux et le plus redouté pour un matériau fragile (1). Il est caractérisé par louverture directe des fissures qui se développent à partir des défauts existants, fissures intra ou intercristallines, cavités, lacunes ou contacts imparfaits entre agrégats et liants.

La résistance à la traction reste cependant l'une des caractéristiques des moins bien définies, notamment à cause de l'absence d'une technique expérimentale qui soit à la fois économique et fiable tout en restant parfaitement rigoureuse au point de vue mécanique.

\section{DÉTERMINATION EXPÉRIMENTALE}

Les nombreuses techniques utilisées (4) pour détermi- ner cette résistance à la traction sont regroupées en quatre catégories : traction homogène, fendage, expansion et flexion.

La multiplicité des méthodes de mesure qui ont été proposées à côté de la simplicité de l'essai de compression montre qu'on n'a pas trouvé d'essai de traction véritablement satisfaisant. La grande dispersion des valeurs expérimentales obtenues liée à l'effet d'échelle rend délicate toute interprétation. Il est important d'examiner en détail les conditions d'essai et d'étudier leur influence sur les valeurs mesurées :

a. L'essai classique de traction directe donne des résultats très dispersés pour certains à cause des difficultés de fixation des éprouvettes (fig. 1). Les têtes de traction doivent être articulées correctement pour éviter les effets de flexion parasites. Le frettage plus marqué pour les éprouvettes encastrées peut conduire à des déformations latérales plus faibles. Du fait de toutes ces précautions, l'essai classique de traction simple est coûteux.
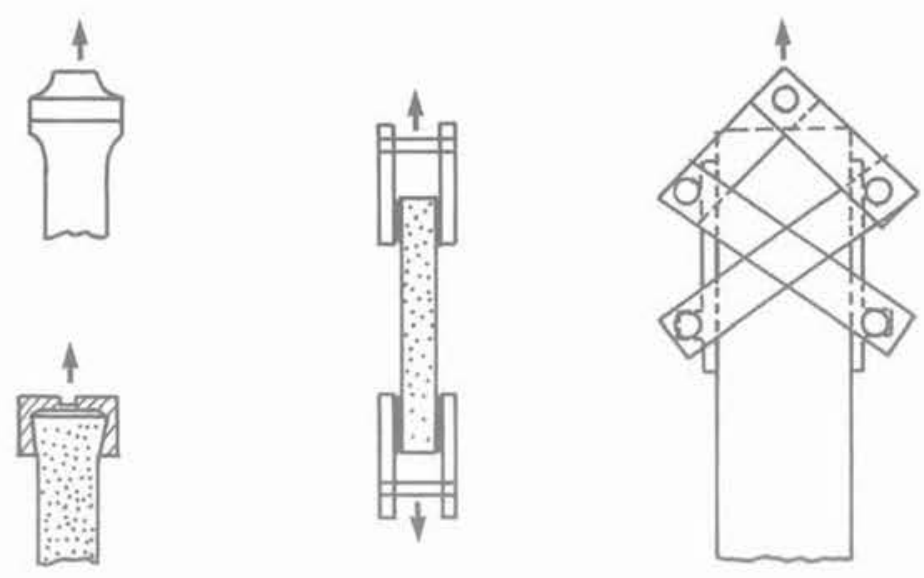

(Morlier)

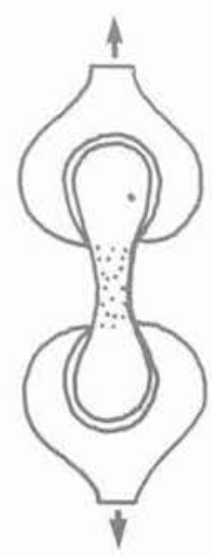

(Michaelis)

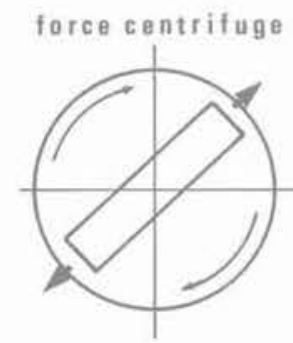

(Berthier)

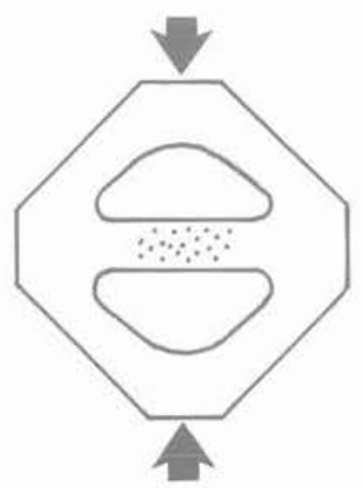

(Desavi \& Veerappan)

Fig. 1. - Traction homogène. 
b. Les essais de fendage (fig. 2) présentent des états de contrainte composés (traction et compression). L'essai brésilien donne des résultats relativement peu dispersés, mais ne peut décrire correctement un comportement autre qu'élastique fragile (2). Les résultats obtenus par fendage donnent des résistances qui peuvent être jusqu'à $50 \%$ plus grands que les essais de traction simple (5). Une plastification inévitable tend à favoriser une redistribution des contraintes, qui entraîne, à son tour, une réduction des niveaux de contraintes là où elles sont les plus élevées. Ce qui a pour effet de retarder la rupture et pourrait être une raison majeure à la surestimation de la résistance à la traction.

c. Les essais en flexion (fig. 3) et en expansion (fig. 4) ont l'inconvénient de présenter des états de contrainte non homogènes. La valeur expérimentale correspond à l'initiation de la rupture du matériau en un point fortement sollicité.
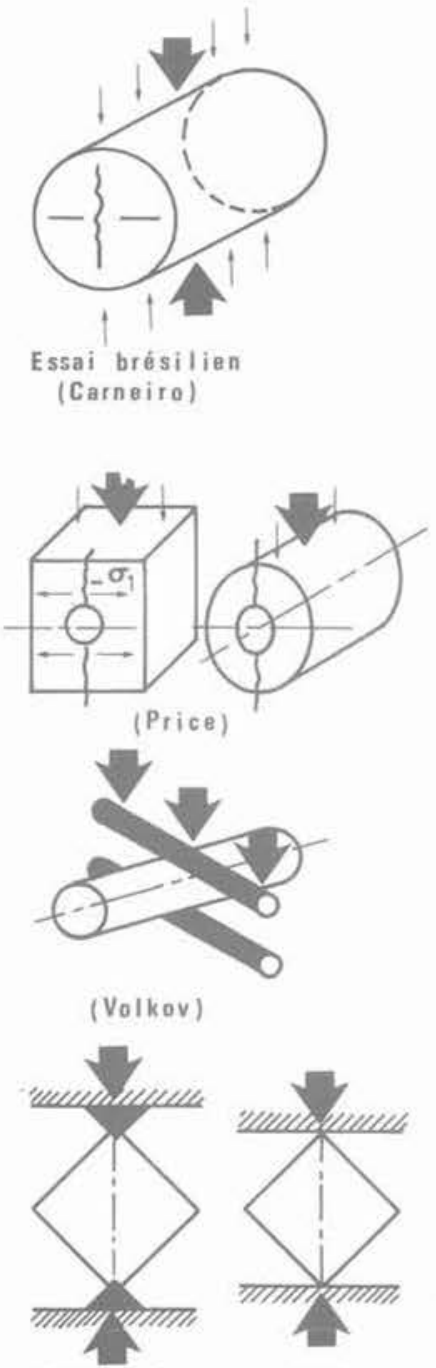

(Dantine)
(Volkov)

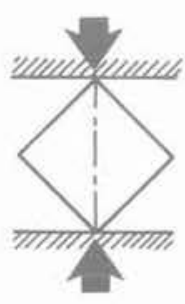

(Sen)
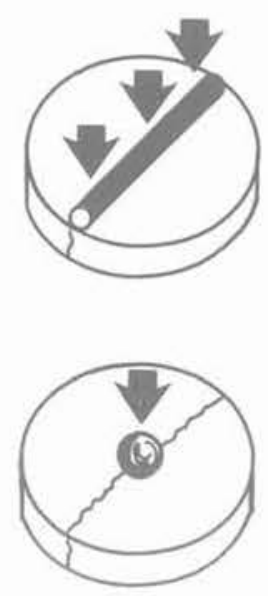

(Paulmann)

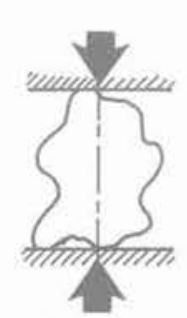

(Protodiakonov) (Hiramatsu)

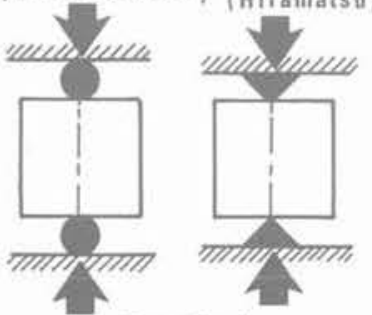

(Berthier)
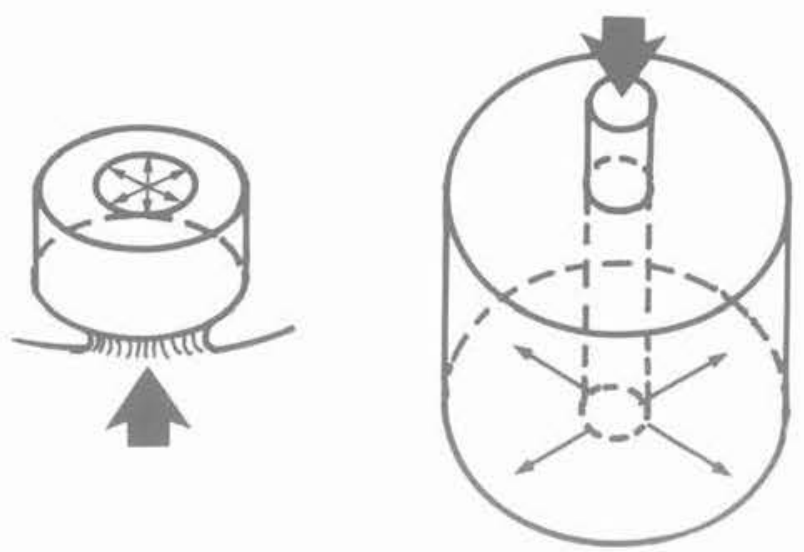

Fig. 4. - Traction par expansion.

d. Une forme bien choisie de l'éprouvette assure une relative homogénéité des déformations, mais elle ne doit pas être trop compliquée à confectionner.

e. La dimension des éprouvettes doit être à une échelle compatible avec les discontinuités du matériau étudié (dimension des agrégats, des fissures et des microfissures).

f. Le dispositif de chargement doit permettre le contrôle de sollicitation en vitesse de charge, vitesse de déplacement ou vitesse de déformation si on désire mettre en évidence le comportement rhéologique avant et après le pic de contrainte.

Fig. 2. - Traction par fendage. 


\section{NOUVEL ESSAI DE TRACTION}

La traction directe sur un matériau est théoriquement le mode le plus simple pour déterminer la résistance à la traction, mais comme on l'a vu plus haut, sa réalisation pratique est délicate. La nouvelle éprouvette d'essai proposée est un tube cylindrique confectionné comme le décrit la figure 5. L'usinage est très facile : deux coups de scie pour les extrémités et deux forages tubulaires coaxiaux inversés; la partie extérieure ne nécessite pas de soin particulier. La configuration proposée réalise une inversion de la sollicitation appliquée sur le corps d'éprouvette, ce qui permet d'utiliser une banale presse de compression. Du fait de la symétrie cylindrique, l'essai ne nécessite pas de dispositif spécial pour la fixation de l'éprouvette et par exemple on peut appliquer les efforts de compression sur les têtes sans précautions spéciales.

L'essai se prête particulièrement bien à des conditions d'environnement hostile : expériences sous haute température, essais de longue durée (fluage ou relaxation), en condition immergée, sous forte pression, sous irradiation, ou pour la résistance aux chocs, etc. Il est aussi possible d'utiliser la même forme d'éprouvette pour des

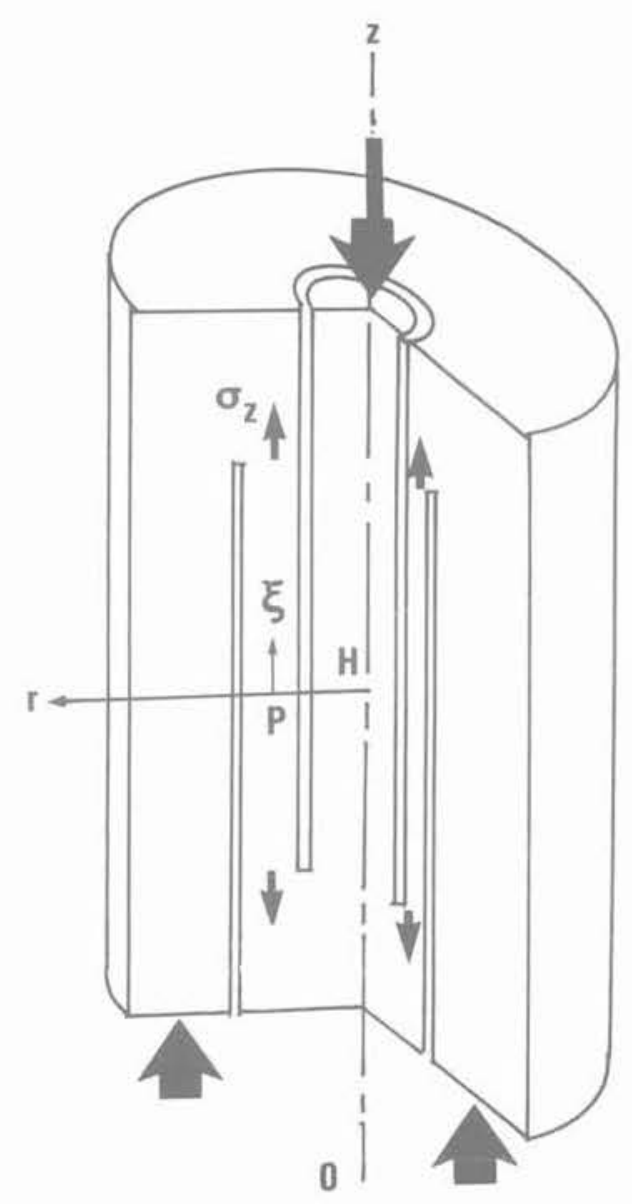

Fig. 5. - Traction directe sur éprouvette tubulaire. essais de compression simple et pour des essais de torsion.

Dans le cas d'un matériau élastique, homogène, isotrope et à l'état initial naturel, le principe de SaintVenant permet de s'assurer qu'à une distance $\mathrm{d}=\pi \sqrt{\mathrm{De}}(\mathrm{D}$ désignant le diamètre du tube, $\mathrm{e}$ son épaisseur), les forces extérieures, s'exerçant dans la section droite du tube, n'interviennent pratiquement que par leurs éléments de réduction. Dans la partie centrale, la distribution des déformations est pratiquement indépendante de 2 . Dès lors, les sections droites restent planes et parallèles (3) :

$$
\varepsilon_{z 2}=\frac{\partial \zeta}{\partial z}=\mathrm{c}=\text { constante }
$$

La composante radiale du déplacement ne peut dépendre que de $r$, soit la solution

$$
\xi=\mathrm{xf}(\mathrm{r}) \quad \eta=\mathrm{yf}(\mathrm{r}) \quad \zeta=\mathrm{cz}
$$

Il y a fonction de déplacement car

$$
\xi d x+\eta d y+\zeta d z=r f(r) d r+c z d z
$$

est une différentielle totale.

Les équations de Lamé et Clapeyron sont satisfaites si la dilatation cubique $\theta$ est constante.

$$
\begin{array}{ll}
\text { D'où } & 2 f+r \frac{d f}{d r}=2 a \\
\text { Soit } & f=a+\frac{b}{r^{2}}(a, b=\text { constantes }) \\
\text { et } & \theta=2 a+c
\end{array}
$$

Les directions principales en un point $\mathrm{P}$ sont donc celles du rayon vecteur $\mathrm{HP}$, de la perpendiculaire au plan méridien $\mathrm{POz}$ et de la parallèle à $\mathrm{Oz}$. Les dilatations suivant ces trois directions sont respectivement :

$$
\varepsilon_{\mathrm{r}}=\mathrm{a}-\frac{\mathrm{b}}{\mathrm{r}^{2}} \quad \varepsilon_{0}=\mathrm{a}+\frac{\mathrm{b}}{\mathrm{r}^{2}} \quad \varepsilon_{\mathrm{z}}=\mathrm{c}
$$

Dans le cas de la traction simple sur les extrémités, on a :

$$
\sigma_{\mathrm{r}}=0 \quad \sigma_{\theta}=0 \quad \sigma_{z}=\mathrm{F} / \pi\left(\mathrm{r}_{\mathrm{e}}^{2}-\mathrm{r}_{\mathrm{i}}^{2}\right)
$$

( $r_{e}$ et $r_{i}$ sont respectivement les rayons extérieur et intérieur), ce qui donne:

$$
\begin{aligned}
& 2(\lambda+\mu) \mathrm{a}+\lambda \mathrm{c}=0 \\
& \mathrm{~b}=0 \text { et } 2 \lambda \mathrm{a}+(\lambda+2 \mu) \mathrm{c}=\mathrm{F} / \pi\left(\mathrm{r}^{2}-\mathrm{r}^{2}\right)
\end{aligned}
$$

Un calcul par éléments finis avec le programme du L.M.S. mis au point par Y. MÉZIÊRE a permis d'optimiser la forme de l'éprouvette (fig. 6). 


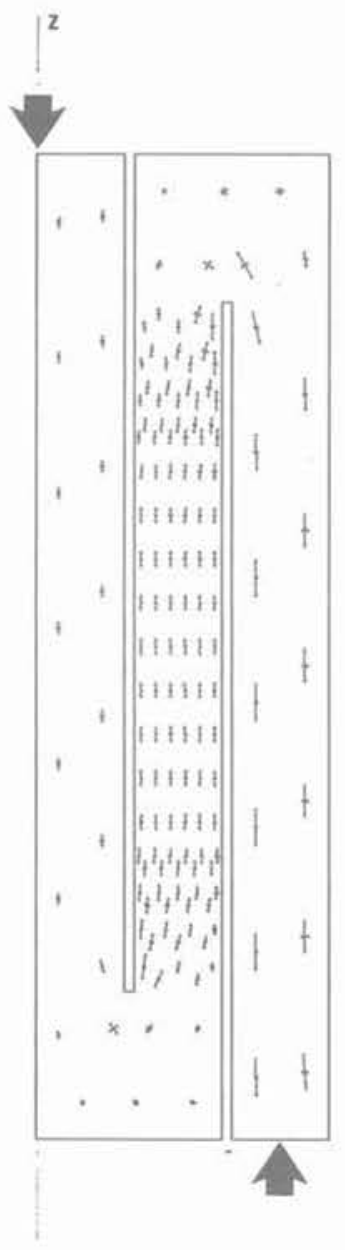

Fig. 6. - Contraintes principales dans une section méridienne de l'éprouvette.

\section{RÉSULTATS D'ESSAIS}

Nous donnons ci-dessous des résultats d'essais obtenus sur une série de mesures de la résistance à la traction du béton. Les éprouvettes ont été prélevées dans des cylindres de dimensions $\varnothing=16 \mathrm{~cm}$ et $\mathrm{h}=32 \mathrm{~cm}$ fabriqués par le CES-CEBTP à la composition suivante :

- Gravier silico-calcaire de Seine (5 - 12,5 mm) $10,8 \mathrm{kN} / \mathrm{m}^{3}$ de béton frais

- Sable de Seine (0 - $5 \mathrm{~mm})$

$6,8 \mathrm{kN} / \mathrm{m}^{3}$ de béton frais

- Ciment CPA 400 prise de mer $4,0 \mathrm{kN} / \mathrm{m}^{3}$ de béton frais

- Eau 220 litres $/ \mathrm{m}^{3}(\mathrm{E} / \mathrm{C}=0,55)$

Décoffrés à deux jours d'ầge, les cylindres de béton ont été conservés dans des conditions thermohygrométriques contrôlées.
Les caractéristiques mécaniques déterminées à trois mois dans l'air sont :

- Résistance en compression simple

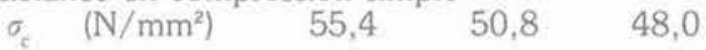

- Module d'élasticité initial instantané

$$
\mathrm{E}_{\mathrm{bj}}\left(\mathrm{kN} / \mathrm{mm}^{2}\right) \quad 32,4 \quad 33,0 \quad 33,0
$$

- Résistance en traction par un essai de fendage (essai brésilien)

$$
\sigma_{f}\left(\mathrm{~N} / \mathrm{mm}^{2}\right) \quad 4,2 \quad 4,1 \quad 3,9
$$

Un essai de contrôle à deux ans d'âge en compression simple a donné une valeur de $55,9 \mathrm{~N} / \mathrm{mm}^{2}$ avec un module égal à $36,4 \mathrm{kN} / \mathrm{mm}^{2}$.

Les résultats de cinquante-deux essais sur des échantillons tubulaires aux dimensions optimisées à $24 \mathrm{~mm}$ de diamètre extérieur et $17 \mathrm{~mm}$ de diamètre intérieur (fig. 7) ont donné une résistance à la traction simple moyenne de $3,06 \mathrm{~N} / \mathrm{mm}^{2}$ avec un écart-type égal à $0,45 \mathrm{~N} / \mathrm{mm}^{2}$.

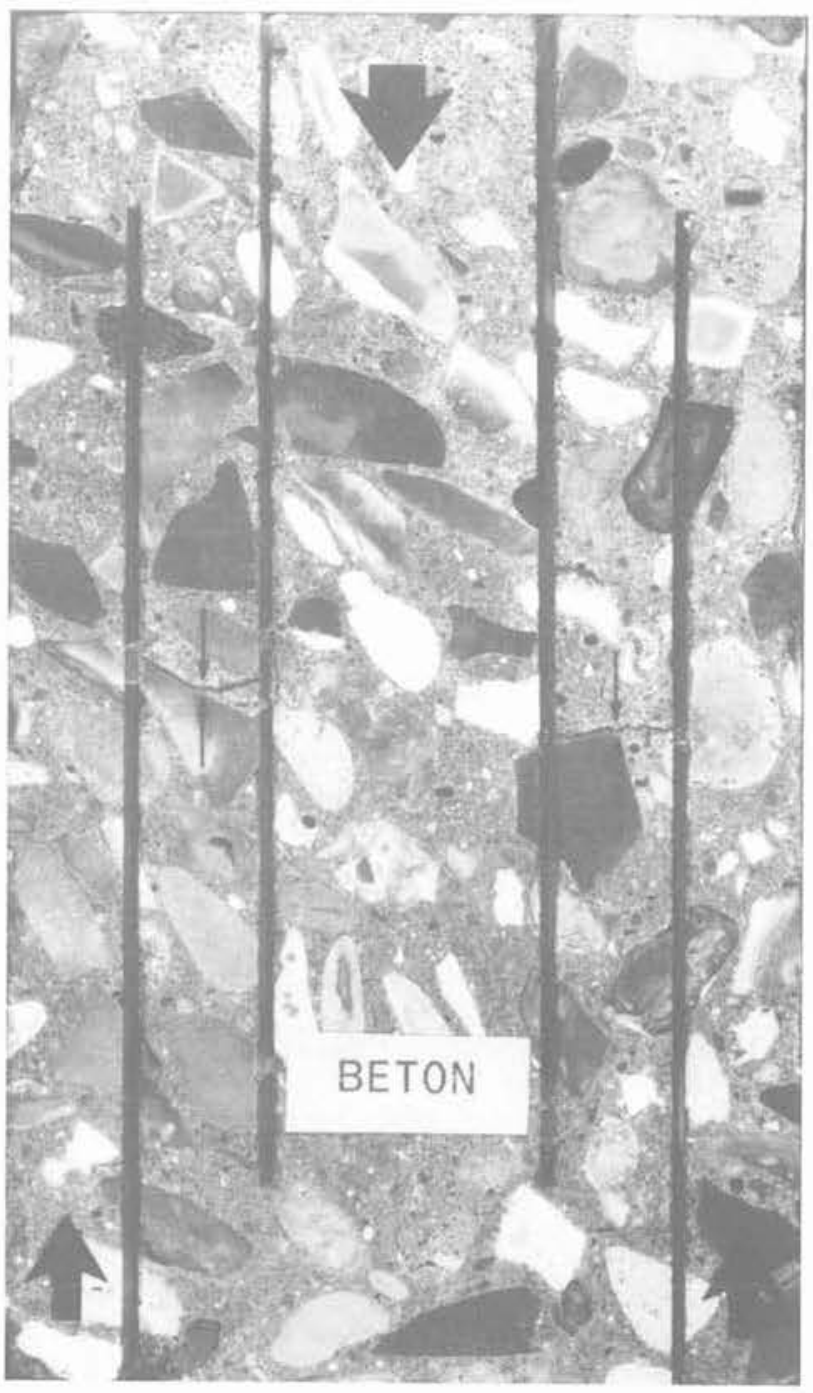

Fig. 7. - Eprouvette de béton en traction. 


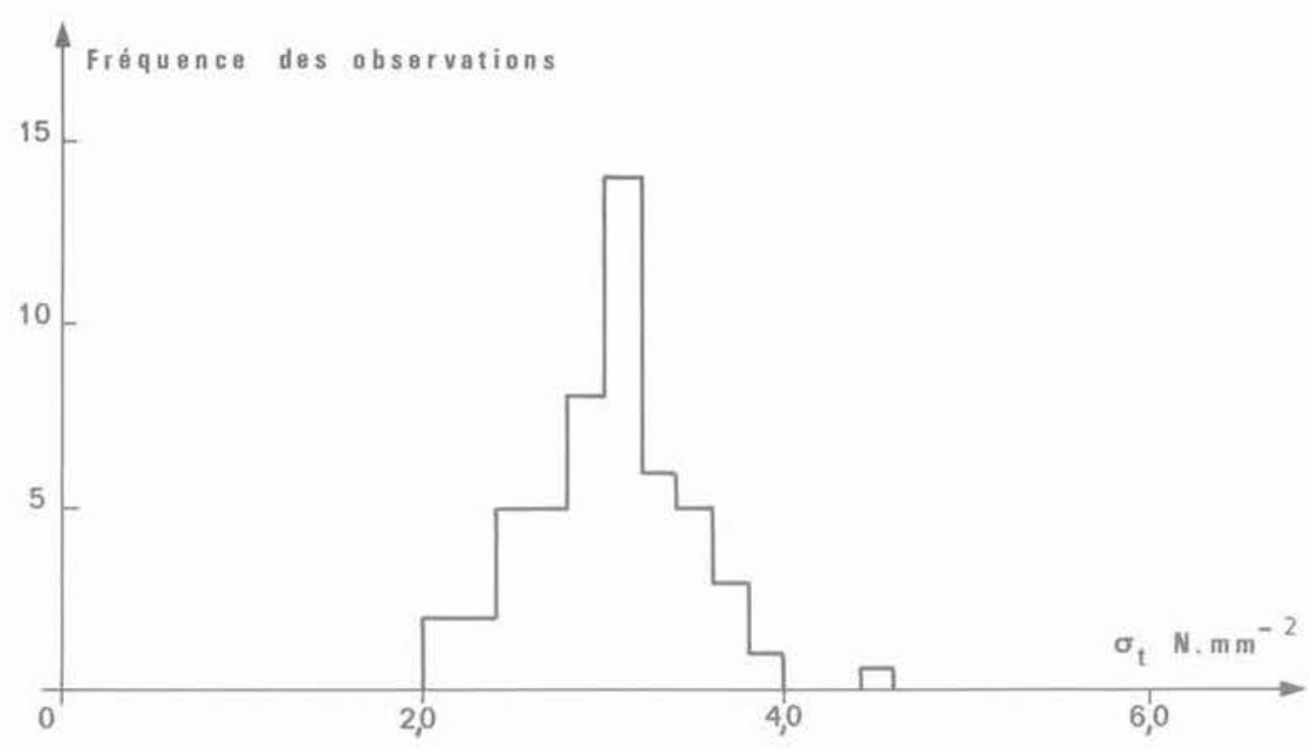

Fig. 8. Histogramme des résultats de 52 essais de traction.

L'histogramme des observations est donné par la figure 8. D'une façon générale les ruptures se sont toujours produites dans la moitié centrale de la partie tubulaire en traction. Des résultats analogues ont été obtenus sur différents matériaux minéraux dont des roches sédimentaires ou métamorphiques.

\section{CONCLUSION}

Le nouvel essai de traction qui a été mis au point au Laboratoire de Mécanique des Solides est d'une réalisation simple, c'est-à-dire qu'il est rapide et économique. Son principe est très satisfaisant sur le plan théorique. Son application aux roches, aux bétons, aux céramiques, aux monocristaux, etc., est aisée et particulièrement commode dans des environnements variés et même hostiles.

\section{BIBLIOGRAPHIE}

1. BUI H.D. (1978), Mécanique de la rupture fragile, Masson, Paris.

2. HABIB P., MORLIER $P$, et RADENKOVIC D. (1964), L'application de l'essai brésilien dans la mécanique des roches, Symposium Rheology and Soil Mechanics, Grenoble, avril 1964.

3. MANDEL J. (1966), Cours de Mécanique des Milieux Continus, tome II, Mécanique des Solides, Gauthier-Villars, Paris.

4. TOURENQ C. et DENIS A. (1970), La résistance à la traction des roches, L.C.P.C. Rapport de recherche ${ }^{\circ} 4$, février 1970, Paris.

5. WRIGHT P.J.F. (1955), Comments on an Indirect Tensile Test on Concrete Cylinders, Magazine of Concrete Research, vol. 7, n⿳20, pp. 87-96. 